gradients on echocardiograms performed during these admissions were 23 and $26 \mathrm{~mm} \mathrm{Hg}$, respectively (Figure 1,C). Unfortunately, the patient died shortly thereafter from complications related to diabetes.

\section{Discussion}

Although this experience is limited to a single case, a low-dose rt-PA regimen with longer infusion duration is a promising approach for patients who are in hemodynamically stable condition and do not require rapid lysis. There may be a lower complication rate because of the significantly lower infusion rate, despite longer infusion duration. Patients should be monitored closely for adequate lysis. This includes daily echocardiography and laboratory evaluations (D-dimer, activated partial thromboplastin time, and fibrinogen). Fluoroscopy should be used to confirm leaflet motion. Further investigation is necessary to evaluate the short- and long-term efficacy and safety of this treatment strategy.

\section{References}

1. Lengyel M, Fuster V, Keltai M, Roudaut R, Schulte HD, Seward JB, et al. Guidelines for management of left-sided prosthetic valve thrombosis: a role for thrombolytic therapy. Consensus Conference on Prosthetic Valve Thrombosis. J Am Coll Cardiol. 1997;30:1521-6.

2. Bonow RO, Carabello BA, Chatterjee K, et al, American College of Cardiology, American Heart Association Task Force on Practice Guidelines (Writing Committee to revise the 1998 guidelines for the management of patients with valvular heart disease), Society of Cardiovascular Anesthesiologists. ACC/AHA 2006 guidelines for the management of patients with valvular heart disease: a report of the American College of Cardiology/American Heart Association Task Force on Practice Guidelines (writing Committee to Revise the 1998 guidelines for the management of patients with valvular heart disease) developed in collaboration with the Society of Cardiovascular Anesthesiologists endorsed by the Society for Cardiovascular Angiography and Interventions and the Society of Thoracic Surgeons [published erratum appears in Circulation. 2007; 115:e409]. Circulation. 2006;114:e84-231.

3. Caceres-Loriga FM, Perez-Lopez H, Santos-Gracia J, MorlansHernandez K. Prosthetic heart valve thrombosis: pathogenesis, diagnosis and management. Int J Cardiol. 2006;110:1-6.

4. Manteiga R, Carlos Souto J, Altès A, Mateo J, Arís A, Dominguez JM, et al. Short-course thrombolysis as the first line of therapy for cardiac valve thrombosis. J Thorac Cardiovasc Surg. 1998;115:780-4.

5. Ozkan M, Kaymaz C, Kirma C, Sönmez K, Ozdemir N, Balkanay M, et al. Intravenous thrombolytic treatment of mechanical prosthetic valve thrombosis: a study using serial transesophageal echocardiography. J Am Coll Cardiol. 2000;35:1881-9.

\title{
Arterial coronary revascularization failure as a result of coronary vasospasm
}

\author{
Marek Gwozdziewicz, MD, PhD, ${ }^{a}$ Martin Sluka, MD, ${ }^{\mathrm{b}}$ and Marek Richter, MD, ${ }^{\mathrm{b}}$ Olomouc, Czech Republic
}

$\mathrm{T}$ otal arterial revascularization (TAR) is an option for the surgical treatment of ischemic heart disease. ${ }^{1}$ It provides better long-term patency than do venous aortocoronary bypasses. TAR seems to offer the most benefit to the patient when combined with the off-pump "aorta no-touch" technique. ${ }^{2}$

Kobayashi and collegues ${ }^{3}$ described a TAR technique that comprises two in situ internal thoracic arteries (ITAs) and a radial artery (RA) used as an I composite graft. They reported that to avoid the competition phenomenon in the graft, the distal end of the I composite should be placed on the right coronary artery (RCA) when this exhibits at least $80 \%$ stenosis.

From the Departments of Cardiac Surgery ${ }^{\mathrm{a}}$ and Cardiology, ${ }^{\mathrm{b}}$ University Hospital, Olomouc, Czech Republic.

Received for publication Sept 26, 2007; accepted for publication Nov 15, 2007.

Address for reprints: Marek Gwozdziewicz, MD, PhD, University Hospital Olomouc, I. P. Pavlova 6, 77515 Olomouc, Czech Republic (E-mail: gwozdziewicz@email.cz).

J Thorac Cardiovasc Surg 2008;135:693-4

$0022-5223 / \$ 34.00$

Copyright $@ 2008$ by The American Association for Thoracic Surgery doi:10.1016/j.jtcvs.2007.11.029

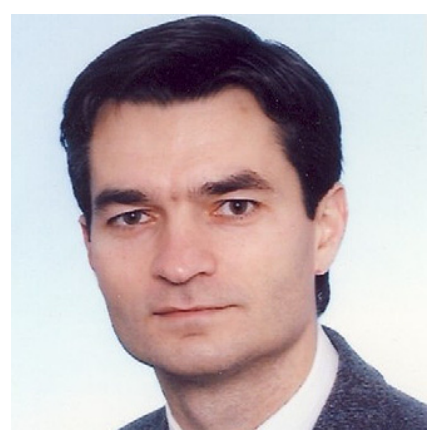

Dr Gwozdziewicz

\section{Clinical Summary}

A 76-year-old man was admitted to our department for elective coronary surgery. Preoperative angiography revealed triple-vessel disease with $40 \%$ left main coronary artery stenosis, $90 \%$ left anterior descending coronary artery stenosis, $90 \%$ diagonal artery stenosis, $80 \%$ circumflex artery stenosis, and at least $80 \%$ proximal RCA stenosis (Figure 1). Echocardiography showed good left ventricular function with no valve disease. The patient had extensive varicose veins on both lower extremities and negative results of an Allen test on both hands. A Doppler examination revealed RAs of good quality with adequate collateral flow.

Quadruple off-pump coronary bypass grafting was performed with the left anterior descending coronary artery bypassed individually by the left ITA. The diagonal artery, obtuse marginal artery, 


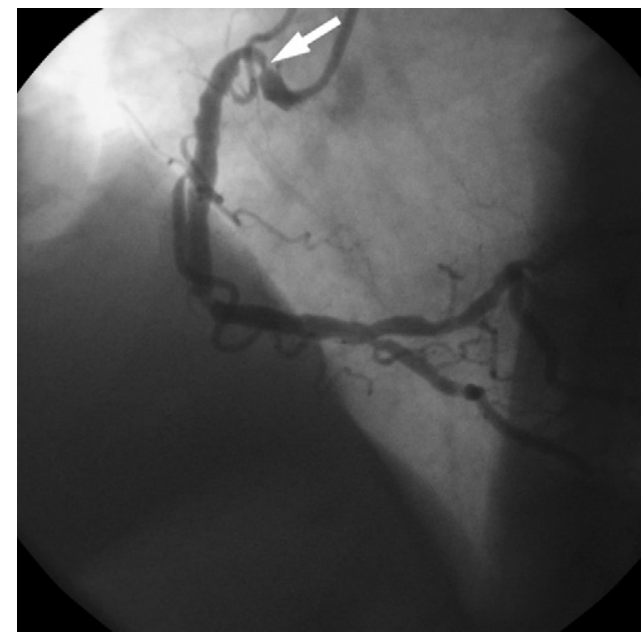

Figure 1. Preoperative coronary angiogram showing at least $\mathbf{8 0} \%$ proximal right coronary artery stenosis (arrow).

and posterior descending branch of the RCA were sequentially grafted with a crossover I composite composed of the right ITA and left RA. Perioperative flow measurement with a transit-time flow meter showed blood flows of 27 and $71 \mathrm{~mL} / \mathrm{min}$ in the left and right ITAs, respectively, with respective pulsatility indexes of 1.7 and 1.4 .

The postoperative course of the patient was uneventful. Postoperative coronary angiography, performed on postoperative day 6 as part of the study protocol, revealed competitive flow in the distal segment of the I composite (Figure 2). All sutured anastomoses were patent. There was $40 \%$ stenosis in the proximal RCA, in contrast to at least $80 \%$ on preoperative angiography.

\section{Discussion}

Most coronary arteries onto which bypass conduits are sutured are not completely occluded and maintain some native blood flow. Sometimes the flow competition between a native artery and a bypass conduit may cause reversal of blood flow in the sutured graft, leading to its closure. This phenomenon has been especially noted in arterial bypass. ${ }^{4}$

TAR with composite grafts is becoming more popular, but many surgeons are concerned about blood flow competition when such grafts are placed in the right coronary system. Kobayashi and colleagues ${ }^{3}$ attempted to solve the problem of competition by suturing the distal end of a sequential I composite graft composed of the right ITA and RA to the RCA when the RCA exhibited at least $80 \%$ stenosis. For stenosis of the RCA less than $80 \%$, they recommended grafting the RCA as the first in the sequence, side-to-side with the composite bypass. This graft orientation should decrease the risk of competition by maintaining the highest blood pressure wave in the anastomosis with the RCA.

Despite our adherence to the recommended rules, our patient had postoperative blood flow competition. This was caused, however, by a coronary vasospasm in the RCA that was misinterpreted as severe stenosis on the preoperative angiogram.

Our experience in this case demonstrates that it is critical for a surgeon who plans to perform the TAR with composite grafts to be aware of the possibility of coronary spasm, leading to overestimation of the degree of stenosis. This is especially important when the lesion is located in the proximal segment of the RCA, where spasm may be provoked by catheter insertion during coronary angiography.

\section{References}

1. Tatoulis J, Buxton BF, Fuller JA, Royse AG. Total arterial coronary revascularization: techniques and results in 3,220 patients. Ann Thorac Surg. 1999;68:2093-9.

2. Lev-Ran O, Braunstein R, Sharony R, Kramer A, Paz Y, Mohr R, et al. No-touch aorta off-pump coronary surgery: the effect on stroke. J Thorac Cardiovasc Surg. 2005;129:307-13.

3. Kobayashi J, Tagusari O, Bando K, Niwaya K, Nakajima H, Ishida M, et al. Total arterial off-pump coronary revascularization with only internal thoracic artery and composite radial artery grafts. Heart Surg Forum. 2002;6:30-7.

4. Nakajima H, Kobayashi J, Tagusari O, Bando K, Niwaya K, Kitamura S. Competitive flow in arterial composite grafts and effect of graft arrangement in off-pump coronary revascularization. Ann Thorac Surg. 2004;78: 481-6.
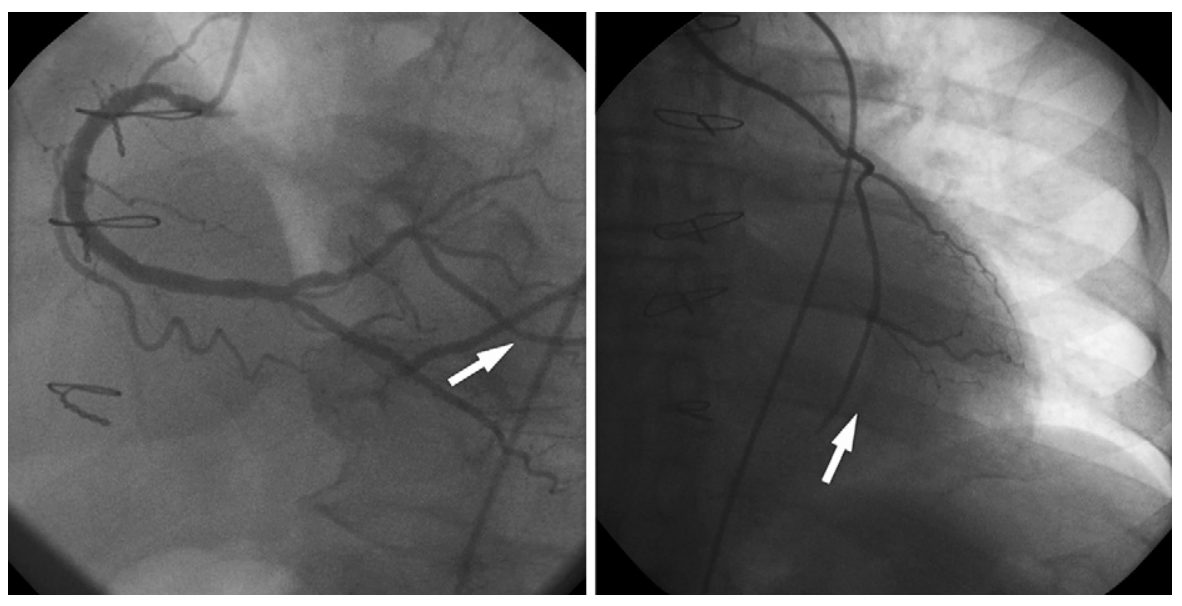

Figure 2. Postoperative coronary angiogram showing competitive flow in distal segment of I composite graft (arrows). 